\title{
Video capsule endoscopy completion and total transit times are similar with oral or endoscopic delivery
}

Authors

Institutions
Peter P. Stanich ${ }^{1}$, John Guido², Bryan Kleinman ${ }^{2,3}$, Kavita Betkerur ${ }^{4,5}$, Kyle M. Porter ${ }^{6}$, Marty M. Meyer ${ }^{1}$

Institutions are listed at the end of article. submitted 14. July 2015 accepted after revision 23. November 2015

\section{Bibliography}

DOI http://dx.doi.org/

10.1055/s-0041-110770

Published online: 15.1.2016

Endoscopy International Open 2016; 04: E228-E232

(c) Georg Thieme Verlag KG Stuttgart · New York

E-ISSN 2196-9736

\section{Corresponding author} Marty M. Meyer, MD 395 W 12th

Ave, Suite 200

Columbus, $\mathrm{OH} 43210$

Phone: +1-614-293-4191

Fax: +1-614-293-8518

Marty.Meyer@osumc.edu
Background and study aims: Video capsule endoscopy (VCE) is limited by incomplete procedures. There are also contraindications to the standard ingestion of the capsule that require endoscopic placement. Our aim was to compare the study completion rate of VCE after oral ingestion and endoscopic deployment.

Patients and methods: We performed a review of all VCE from April 2010 through March 2013. Inpatient and outpatient cohorts grouped by the method of capsule delivery were formed and compared. Multivariable logistic regression modeling was utilized adjusting for variables with a $P$ value $\leq 0.1$ in group comparisons. Log-rank analysis was used to compare transit times.

Results: A total of 687 VCE were performed, including 316 inpatient (36 endoscopic deploy-

\section{Introduction}

$\nabla$

Since its introduction in 2000 , video capsule endoscopy (VCE) has become the test of choice for direct visualization of the small bowel [1]. The VCE is generally swallowed and then native bowel motility is utilized for propulsion of the device through the gastrointestinal tract. Complete examination, defined as the VCE traversing the entirety of the small bowel and reaching the colon during battery life, is accomplished in $83.5 \%$ of VCE procedures [2].

Because oral ingestion of the device may not be suitable for some patients, such as those with oropharyngeal dysphagia or esophageal stricture, methods have been developed to place the VCE directly into the small bowel with endoscopic delivery $[3,4]$. It has also been suggested that endoscopic delivery of the VCE may increase completion rates as delayed gastric transit has been identified as a risk factor for incomplete procedures $[5,6]$. There is also concern that the sedation used for endoscopy will lead to decreased motility and incomplete exams. The available evidence ment, 280 oral ingestion) and 371 outpatient (20 endoscopic deployment, 351 oral ingestion). For VCE on hospitalized patients, the completion rates were similar after endoscopic deployment and oral ingestion ( $72 \%$ vs $73 \%, P=0.94)$. The completion rates were also similar for ambulatory patients ( $90 \%$ vs $87 \%, P=0.69$ ). There remained no difference after multivariable modeling for inpatients $(P=0.71)$ and outpatients $(P=0.46)$. Total transit times were not significantly different.

Conclusions: VCE completion rates and total transit times are similar after oral or endoscopic deployment for both hospitalized and ambulatory patients. Endoscopic placement is effective in patients with contraindications to standard oral ingestion, but should otherwise be avoided to limit unnecessary procedural risks and costs.

on the utility of endoscopic placement is limited and conflicting [7-9].

The primary clinical aim of our study was to clarify the outcomes of VCE placed endoscopically, especially given the importance of VCE completion to avoid missing lesions or performing unnecessary repeated exams. To accomplish this, we investigated our experience with endoscopic deployment of VCE for hospitalized and ambulatory patients with a primary focus on completion rate and bowel transit times.

\section{Patients and methods \\ $\nabla$}

\section{Patients}

The appropriate Institutional Review Board at the Ohio State University approved this study prior to initiation. A database of all patients aged 18 years or older undergoing VCE at our institution from April 2010 through March 2013 was utilized for analysis. It was compiled retrospectively and contained demographic data and information on clinical characteristics including medical history 


\begin{tabular}{|c|c|c|c|}
\hline & Endo & Oral & $P$ value $^{1}$ \\
\hline & $n=36$ & $n=280$ & \\
\hline Sex (female), n (\%) & $23(64 \%)$ & $168(60 \%)$ & 0.65 \\
\hline Age, mean (SD) & $63(14)$ & $62(15)$ & 0.62 \\
\hline Body mass index, mean (SD) & $22(26)$ & $30(8)$ & 0.11 \\
\hline Bowel preparation, $\mathrm{n}(\%)$ & $36(100 \%)$ & $278(99 \%)$ & 1 \\
\hline Poor preparation, n (\%) & $2(6 \%)$ & $24(9 \%)$ & 0.53 \\
\hline Patency capsule performed, $\mathrm{n}(\%)$ & $5(14 \%)$ & $44(16 \%)$ & 0.78 \\
\hline Total hospital days, $\mathrm{n}(\mathrm{SD})$ & $14(17)$ & $11(8)$ & 0.22 \\
\hline VCE performed on hospital day, $n$ (SD) & $9(15)$ & $6(4)$ & 0.44 \\
\hline \multicolumn{4}{|l|}{ Clinical characteristics } \\
\hline Small bowel surgery, $\mathrm{n}(\%)$ & $5(14 \%)$ & $43(15 \%)$ & 0.82 \\
\hline Bariatric surgery, $\mathrm{n}(\%)$ & $4(11 \%)$ & $11(4 \%)$ & 0.06 \\
\hline History of bowel obstruction, $\mathrm{n}(\%)$ & $0(0 \%)$ & $11(4 \%)$ & 0.62 \\
\hline Diabetes mellitus, $\mathrm{n}(\%)$ & $13(36 \%)$ & $122(44 \%)$ & 0.39 \\
\hline Crohn's disease, $n(\%)$ & $0(0 \%)$ & $15(5 \%)$ & 0.23 \\
\hline Systemic disease affecting motility, $\mathrm{n}(\%)^{2}$ & $8(22 \%)$ & $56(20 \%)$ & 0.75 \\
\hline \multicolumn{4}{|l|}{ Medications } \\
\hline Opiates, $n(\%)$ & $32(89 \%)$ & $147(53 \%)$ & $<0.001$ \\
\hline Prokinetics, n (\%) & $0(0 \%)$ & $16(6 \%)$ & 0.23 \\
\hline Beta blockers, n (\%) & $16(44 \%)$ & $154(55 \%)$ & 0.23 \\
\hline Calcium channel blockers, n (\%) & $4(11 \%)$ & $52(19 \%)$ & 0.27 \\
\hline Anticholinergics, $\mathrm{n}(\%)$ & $4(11 \%)$ & $39(14 \%)$ & 0.64 \\
\hline Iron supplementation, $\mathrm{n}(\%)$ & $14(39 \%)$ & $72(26 \%)$ & 0.09 \\
\hline VCE indications & & & 0.33 \\
\hline Occult bleeding ${ }^{3}$ & $9(25 \%)$ & $74(26 \%)$ & \\
\hline Overt bleeding & $27(75 \%)$ & $183(65 \%)$ & \\
\hline Crohn's disease & $0(0 \%)$ & $19(7 \%)$ & \\
\hline Small bowel tumors & $0(0 \%)$ & $0(0 \%)$ & \\
\hline Others & $0(0 \%)$ & $4(1 \%)$ & \\
\hline \multicolumn{4}{|l|}{ VCE results } \\
\hline Completed exam, n (\%) & $26(72 \%)$ & $204(73 \%)$ & 0.94 \\
\hline Gastric transit time, min., median (IQR) & & $45(12,110)$ & \\
\hline Small bowel transit time, min., median (IQR) & $290(221,>480)$ & $222(163,349)$ & 0.03 \\
\hline Total transit time, min., median (IQR) & $290(221,>480)$ & $287(203,472)$ & 0.75 \\
\hline
\end{tabular}

Table 1 Patient demographics, clinical characteristics and outcomes after ingestion or endoscopic deployment of video capsule endoscopy in hospitalized patients.

Endo, endoscopic deployment; IQR, inter-quartile range; Min., min; VCE, video capsule endoscopy.

${ }^{1}$ Chi-square or Fisher's exact tests for categorical variables, $t$ tests or Mann-Whitney $U$ test for continuous variables and log rank for transit times.

2 This included hyperthyroidism, hypothyroidism, amyloidosis, scleroderma, connective tissue disease, Parkinson's disease and multiple sclerosis.

${ }^{3}$ Includes iron deficiency anemia.

and medications and VCE indication, results, and delivery method.

For the current study, the method of capsule delivery and association with VCE completion and transit were the primary variables of interest. Distinct cohorts were created based on hospitalization status given the consistent differences in completion rates between ambulatory and hospitalized patients receiving VCE [5, $6,10]$. Within these cohorts, patients were then separated based on method of capsule delivery. Relevant demographics, clinical characteristics and medical history were collected. In addition to the common diseases known to affect bowel motility noted in the tables that were analyzed separately, we assessed less common conditions (thyroid abnormalities, amyloidosis, scleroderma, mixed connective tissue diseases, Parkinson's disease and multiple sclerosis) separately as well as in total as "systemic disease affecting motility."

\section{Procedure}

All VCE during the study period were performed with PillCam SB 2 Video Capsules (Given Imaging, Yoqneam, Israel) and interpreted with the associated software by either of two experienced physicians (including author MMM). Method of capsule delivery was decided upon by the ordering physician's preference. When placed endoscopically, a specialized capsule delivery device was used (AdvanCE delivery device, US Endoscopy, Mentor, Ohio, United States). All endoscopic procedures were performed under standard procedural sedation (conscious sedation with midazolam and a narcotic agent (predominantly fentanyl), monitored anesthesia care or general anesthesia) as determined by the ordering physician. Gastric transit time was calculated by the minutes (min.) that separated the first image of stomach from the first image of duodenum. Small bowel transit time was calculated as the min. that separated the first image of duodenum from the first image of cecum. Total transit time was the combination of these measures for patients with oral ingestion and placement time and small bowel transit time for patients with endoscopic deployment. The small bowel examination was considered to be complete when the cecum was reached during the battery lifespan.

The standard bowel preparation recommended was $2 \mathrm{~L}$ of a polyethylene glycol 3350, sodium bicarbonate, sodium chloride, and potassium chloride bowel purgative solution (Trilyte; Alaven 


\begin{tabular}{|c|c|c|c|}
\hline & Endo & Oral & $P$ value ${ }^{1}$ \\
\hline & $\mathrm{n}=\mathbf{2 0}$ & $n=351$ & \\
\hline Sex (female), n (\%) & $15(75 \%)$ & $218(62 \%)$ & 0.25 \\
\hline Age, mean (SD) & $62(13)$ & $52(17)$ & 0.01 \\
\hline Body mass index, mean (SD) & $29(9)$ & $30(8)$ & 0.69 \\
\hline Bowel preparation, $\mathrm{n}(\%)$ & $17(85 \%)$ & $342(97 \%)$ & 0.002 \\
\hline Poor preparation, n (\%) & $2(10 \%)$ & $27(8 \%)$ & 0.71 \\
\hline Patency capsule performed, $\mathrm{n}(\%)$ & $3(15 \%)$ & $70(20 \%)$ & 0.59 \\
\hline \multicolumn{4}{|l|}{ Total hospital days, $\mathrm{n}(\mathrm{SD})$} \\
\hline \multicolumn{4}{|l|}{ VCE performed on hospital day, n (SD) } \\
\hline \multicolumn{4}{|l|}{ Clinical characteristics } \\
\hline Small bowel surgery, $\mathrm{n}(\%)$ & $2(10 \%)$ & $22(6 \%)$ & 0.51 \\
\hline Bariatric surgery, n (\%) & $2(10 \%)$ & $10(3 \%)$ & 0.24 \\
\hline History of bowel obstruction, n (\%) & $0(0 \%)$ & $11(3 \%)$ & 0.42 \\
\hline Diabetes mellitus, n (\%) & $4(20 \%)$ & $66(19 \%)$ & 0.9 \\
\hline Crohn's disease, n (\%) & $0(0 \%)$ & $31(9 \%)$ & 0.16 \\
\hline Systemic disease affecting motility, $\mathrm{n}(\%)^{2}$ & $3(15 \%)$ & $62(18 \%)$ & 0.97 \\
\hline \multicolumn{4}{|l|}{ Medications } \\
\hline Opiates, n (\%) & $16(80 \%)$ & $89(25 \%)$ & $<0.001$ \\
\hline Prokinetics, n (\%) & $0(0 \%)$ & $5(1 \%)$ & 0.59 \\
\hline Beta blockers, n (\%) & $5(25 \%)$ & $97(28 \%)$ & 0.79 \\
\hline Calcium channel blockers, n (\%) & $1(5 \%)$ & $31(9 \%)$ & 0.55 \\
\hline Anticholinergics, $n(\%)$ & $2(10 \%)$ & $84(24 \%)$ & 0.15 \\
\hline Iron supplementation, n (\%) & $8(40 \%)$ & $104(30 \%)$ & 0.33 \\
\hline VCE indications & & & 0.01 \\
\hline Occult bleeding ${ }^{3}$ & $5(25 \%)$ & $155(44 \%)$ & \\
\hline Overt bleeding & $9(49 \%)$ & $72(21 \%)$ & \\
\hline Crohn's disease & $0(0 \%)$ & $55(16 \%)$ & \\
\hline Small bowel tumors & $0(0 \%)$ & $20(6 \%)$ & \\
\hline Others & $6(30 \%)$ & $49(14 \%)$ & \\
\hline \multicolumn{4}{|l|}{ VCE results } \\
\hline Completed exam, n (\%) & $18(90 \%)$ & $305(87 \%)$ & 0.69 \\
\hline Gastric transit time, min., median (IQR) & & $27(14,64)$ & \\
\hline Small bowel transit time, min., median (IQR) & $229(165,352)$ & $212(139,284)$ & 0.56 \\
\hline Total transit time, min., median (IQR) & $229(165,352)$ & $255(180,334)$ & 0.64 \\
\hline
\end{tabular}

Table 2 Patient demographics, clinical characteristics and outcomes after ingestion or endoscopic deployment of video capsule endoscopy in ambulatory patients.

Endo, endoscopic deployment; IQR, inter-quartile range; Min., min; VCE, video capsule endoscopy.

${ }^{1}$ Chi-square or Fisher's exact tests for categorical variables, $t$ tests or Mann-Whitney $\mathrm{U}$ test for continuous variables and log rank for transit times.

${ }^{2}$ This included hyperthyroidism, hypothyroidism, amyloidosis, scleroderma, connective tissue disease, Parkinson's disease and multiple sclerosis.

${ }^{3}$ Includes iron deficiency anemia.

Pharmaceutical LLC, Marietta, GA) the evening before the procedure and no oral intake starting at 12 AM the day of the procedure. Patients were instructed that clear liquid intake could be started at 2 hours after VCE ingestion and a light meal after 4 hours. No prokinetic medications were utilized specifically for the procedure.

\section{Statistical analysis}

Patient demographics and clinical characteristics were presented as means with standard deviations (SD) for continuous variables and as counts and percentages for categorical variables. Group comparisons were made using chi-square or Fisher's exact tests for categorical variables and $t$ tests or Mann-Whitney $U$ tests for continuous variable. Multivariable logistic regression modeling for VCE completion was performed adjusting for variables with a $P$ value $\leq 0.1$ in group comparisons. Median and inter-quartile range (IQR) were used to summarize gastric, small bowel, and total transit times. Log rank testing was performed to compare transit times between delivery methods. A Kaplan-Meier curve was produced to display the total transit times and completion rates of VCE stratified by hospitalization status and delivery method, with data censored at the end of battery life ( $480 \mathrm{~min}$.) if incomplete.

\section{Results}

During the study period, 687 VCE were performed and 56 (12.3\%) were placed endoscopically. The demographics and clinical characteristics of the hospitalized patient cohort and the ambulatory patient cohort information are detailed in $\bullet$ Table1 and - Table 2 respectively. Indications for endoscopic deployment included a history of previous gastric retention of VCE for 20 (36\%), dysphagia for $9(16 \%)$, concomitant upper endoscopy procedure on same date for 8 (14\%), esophageal or gastric surgical history for $6(11 \%)$ and duodenal stricture in $1(2 \%)$. The indication for endoscopic placement was unknown for 12 (21\%) patients. Endoscopic deployment in the small bowel was successful in 54 (96.4\%) patients. One failure was unsuccessful due to persistent pylorospasm that prevented device passage, which result- 
ed in gastric deployment; in the other case, no reasons was stated for the gastric placement. No complications were noted during the procedures or with the use of the delivery device.

A total of 317 inpatient procedures were performed with 36 (11.4\%) of the patients receiving endoscopic deployment. Exam completion rates were similar, with a $72 \%$ completion rate when endoscopically placed and $73 \%$ with oral ingestion $(P=0.94$, - Fig.1). The small bowel transit time was significantly increased after endoscopic deployment $(290 \mathrm{~min}$. vs $222 \mathrm{~min}$., $P=$ $0.03)$. Total transit time, however, was similar between the groups ( $290 \mathrm{~min}$. vs $287 \mathrm{~min}$., $P=0.75$ ). Of note, the endoscopic deployment group had a significant increase in opiate usage within 24 hours of the procedure $(P<0.001)$ and a trend toward a history of bariatric surgery $(P=0.06)$ and use of iron supplementation $(P=0.09)(\bullet$ Table 1$)$. When these were included in multivariable analysis, there remained no difference in VCE complete rate $(P=0.71)$.

A total of 371 outpatient procedures were performed with 20 (5.4\%) using endoscopic placement. Exam completion rates were comparable between endoscopic deployment and oral ingestion ( $90 \%$ vs $87 \%$ respectively, $P=0.69$ ) ( $\odot$ Fig. 1 ). Small bowel and total transit times were also comparable between the groups. The endoscopic deployment group was significantly older (62 years vs 52 years, $P=0.01$ ) and a smaller proportion of the patients had completed bowel preparation ( $85 \%$ vs $97 \%, P=$ 0.002 ). They also had a significant increase in opiate usage within 24 hours of the procedure ( $80 \%$ vs $25 \%, P<0.001$ ) ( $\bullet$ Table 2 ). The indications for VCE were also significantly different between the groups $(P=0.01)(-$ Table 2$)$. When these were included in multivariable analysis, there remained no difference in VCE complete rate $(P=0.46)$.

\section{Discussion}

VCE is the favored method for evaluation of the small bowel mucosa, but given its reliance on bowel motility for propulsion, can result in an incomplete examination. Oral ingestion of the capsule is the standard method for delivery of the device, but endoscopic techniques for direct small bowel delivery have been developed for use when ingestion is contraindicated or when gastric motility is abnormal.

We described remarkably similar completion rates and total transit times independent of delivery method for both hospitalized and ambulatory patients receiving capsule endoscopy. Importantly, this similarity remained after multivariable analysis to account for demographic and clinical differences. Based on our results, oral ingestion should continue to be the preferred method of capsule delivery given the increased risks and costs necessitated by the addition of an endoscopic procedure and sedation.

Our findings also support continued use of endoscopic delivery of VCE when indicated given the equivalent completion rates. This includes patients with oropharyngeal dysphagia or symptomatic esophageal dysphagia, uncontrolled gastroparesis or a history of gastric retention of VCE. Based on our previously reported findings, endoscopic deployment is neither necessary nor beneficial in patients with a history of Roux-en-y gastric bypass or similar post-surgical anatomy [11]. Whether endoscopic placement of the VCE should be completed at the time of an upper endoscopy being performed for other indications is debatable. In our clinical experience, the trauma caused by endoscopy or biopsies in the

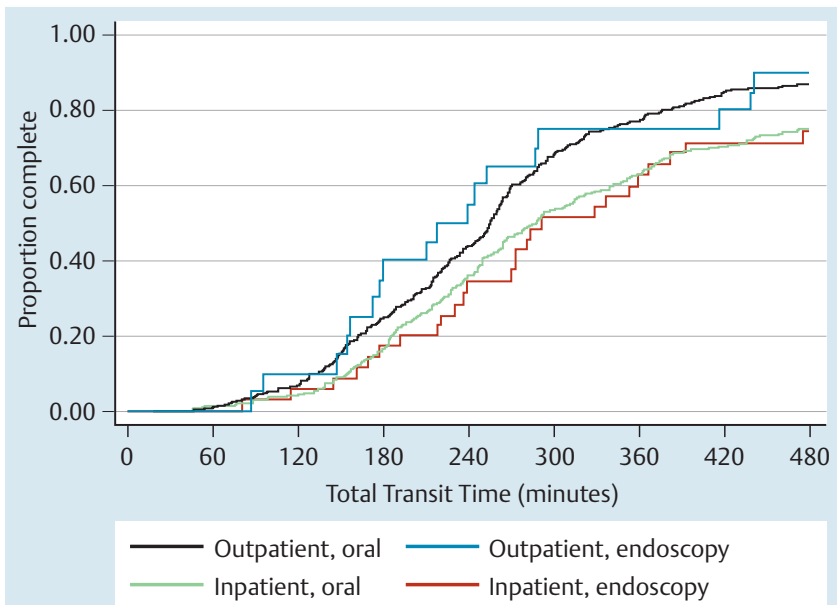

Fig. 1 Analysis of video capsule endoscopy completion grouped by delivery method and hospitalization status. Kaplan-Meier analysis of video capsule endoscopy completion after endoscopic deployment and oral ingestion for both inpatient and outpatient procedures was performed. The endpoint was a completed study and data were censored at the end of battery life ( $480 \mathrm{~min})$. Cox proportional hazards model testing showed no significant difference between the delivery methods for both inpatients $(P=0.75)$ and outpatients $(P=0.64)$.

proximal duodenum can be difficult to delineate from truly pathogenic lesions. In addition, the low risk of a second esophageal intubation with the VCE and delivery device in place must be weighed against the possible benefit of streamlining patient care. This deserves further study, but we currently favor oral ingestion of the VCE once procedural sedation has dissipated and the patient is alert enough to safely take liquids.

There is minimal published data regarding the experience of other centers in this field, and what are available reflect conflicting results. Almeida et al. reported on a small series of completed VCE studies in 10/13 patients (77\%) after endoscopic deployment, but they did not report the hospitalization status of their patients, which is an important potential confounding factor [7]. Gibbs and Bloomfield reported on 33 inpatient and 26 outpatient VCE with endoscopic placement. They had a $64.4 \%$ completion rate overall (66\% for hospitalized patients and $61.5 \%$ for ambulatory patients) [8]. Although they do not report their oral ingestion completion rates for comparison, their experience is markedly lower than the expected completion rates based on systematic review [2]. Both of these studies utilized endoscopic placement for similar indications as were included in our cohorts. In contrast, Matsunaga et al. performed a prospective trial of unsedated transnasal endoscopy for VCE placement that was offered to all patients at their center. They found a significantly increased completion rate ( $83.3 \%$ vs $61.1 \%, P=0.046$ ) in the 24 patients who opted for the procedure and had successful duodenal placement [9]. The avoidance of procedural sedation for the endoscopic placements in this study is likely a factor in their positive findings. However, an important consideration with this study is that three patients had unsuccessful placement into the duodenum and were not included in the analysis. Unfortunately, because the outcomes of these VCE were not reported, an intention-to-treat analysis cannot be performed. If they are assumed to be incomplete, the association would no longer be significant ( $74 \%$ vs $61.1 \%, P=0.25$ ). They also do not report the hospitalization status of the study subjects, which is a potential confounding factor. 
Considering results of these previous studies in conjunction with our findings underscores that endoscopic placement should be reserved for patients with a contraindication to oral ingestion but remains a useful tool when needed. An exciting future option may be the use of a real-time viewer to blend standard oral ingestion with the use of endoscopic placement when needed in order to optimize completion $[12,13]$.

The strength of our study is the inclusion of a large cohort of patients who received VCE at our center during the period studied and the ability to directly compare the results of endoscopic deployment and oral ingestion of the device. Due to this size, we were able to avoid the confounding factor of hospitalization status that limited all previous studies. We were unable to assess the mobility of the hospitalized patients, which may have affected transit times [14]. However, there were only 2 patients in the endoscopically placed group who were in the intensive care unit and there was no mention of strict bed rest orders for the other patients. Standard limitations also apply to clinical research performed retrospectively, including the possibility of selection bias. However, the majority of patients in the endoscopically placed cohorts had clearly stated indications for this modality that fit clinical guidelines and this should lessen that concern.

\section{Conclusions}

$\nabla$

Completion rates and total transit times for VCE are similar after oral or endoscopic deployment in both hospitalized and ambulatory patients. Endoscopic deployment is effective in patients with contraindications to standard oral ingestion, but should otherwise be avoided to limit unnecessary procedural risks and costs.

\section{Competing interests: None}

Institutions

${ }^{1}$ Section of Capsule Endoscopy, Division of Gastroenterology, Hepatology \& Nutrition, The Ohio State University Wexner Medical Center, Columbus, Ohio, United States

${ }^{2}$ Department of Internal Medicine, The Ohio State University Wexner Medical Center, Columbus, Ohio, United States

${ }^{3}$ Division of Gastroenterology; Einstein Medical Center, Philadelphia, Pennsylvania, United States

${ }^{4}$ College of Medicine, The Ohio State University, Columbus, Ohio, United States

${ }^{5}$ Department of Internal Medicine, UC Davis School of Medicine, Sacramento, California, United States

${ }^{6}$ Center for Biostatistics, The Ohio State University, Columbus, Ohio, United States

\section{References}

1 Iddan G, Meron G, Glukhovsky A et al. Wireless capsule endoscopy. Nature 2000; 405: 417

2 Liao Z, Gao R, Xu C et al. Indications and detection, completion, and retention rates of small-bowel capsule endoscopy: a systematic review. Gastrointestinal endoscopy 2010; 71: 280-286

3 Carey EJ, Heigh RI, Fleischer DE. Endoscopic capsule endoscope delivery for patients with dysphagia, anatomical abnormalities, or gastroparesis. Gastrointestinal endoscopy 2004; 59: 423-426

4 Holden JP, Dureja P, Pfau PR et al. Endoscopic placement of the smallbowel video capsule by using a capsule endoscope delivery device. Gastrointestinal endoscopy 2007; 65: 842-847

5 Westerhof J, Weersma RK, Koornstra JJ. Risk factors for incomplete small-bowel capsule endoscopy. Gastrointestinal endoscopy 2009; 69: $74-80$

6 Yazici C, Losurdo J, Brown MD et al. Inpatient capsule endoscopy leads to frequent incomplete small bowel examinations. World journal of gastroenterology: WJG 2012; 18: 5051 - 5057

7 Almeida N, Figueiredo P, Lopes $S$ et al. Capsule endoscopy assisted by traditional upper endoscopy. Revista espanola de enfermedades digestivas: organo oficial de la Sociedad Espanola de Patologia Digestiva 2008; 100: $758-763$

8 Gibbs WB, Bloomfeld RS. Endoscopic deployment of video capsule endoscopy: does it guarantee a complete examination of the small bowel? Gastrointestinal endoscopy 2012; 76: 905-909

9 Matsunaga T, Hashimoto S, Okamoto T et al. Effect of capsule placement with transnasal endoscopy. Digestive endoscopy : official journal of the Japan Gastroenterological Endoscopy Society 2013; 25: 156-159

10 Ben-Soussan E, Savoye G, Antonietti $M$ et al. Factors that affect gastric passage of video capsule. Gastrointestinal endoscopy 2005; 62: 785790

11 Stanich PP, Kleinman B, Porter KM et al. Video Capsule Endoscopy After Bariatric and Gastric Surgery: Oral Ingestion is Associated With Satisfactory Completion Rate. Journal of clinical gastroenterology 2015; 49: $31-33$

12 Gao YJ, Ge ZZ, Chen HY et al. Endoscopic capsule placement improves the completion rate of small-bowel capsule endoscopy and increases diagnostic yield. Gastrointestinal endoscopy 2010; 72: 103-108

13 Cotter J, de Castro FD, Magalhaes J et al. Finding the solution for incomplete small bowel capsule endoscopy. World journal of gastrointestinal endoscopy 2013; 5: 595-599

14 Shibuya T, Mori H, Takeda $T$ et al. The relationship between physical activity level and completion rate of small bowel examination in patients undergoing capsule endoscopy. Intern Med 2012; 51: 997 - 1001 